\title{
Bacilos Gram negativos entéricos y no fermentadores de la glucosa en pacientes con enfermedad periimplante
}

\author{
Jessika Consuegra1 ${ }^{1}$, Sonia Jakeline Gutiérrez ${ }^{1}$, Adriana Jaramillo ${ }^{1,2}$, Ignacio Sanz ${ }^{3}$, Gilberto Olave ${ }^{2}$, \\ Jorge Enrique Soto ${ }^{2}$, Carlos Valencia ${ }^{2}$, Adolfo Contreras ${ }^{1,2}$ \\ 1 Joven Investigadora Univalle-Colciencias, Grupo de Medicina Periodontal, Escuela de Odontología, Universidad \\ del Valle, Cali, Colombia \\ 2 Departamento de Periodoncia, Escuela de Odontología, Universidad del Valle, Cali, Colombia \\ 3 Departamento de Periodoncia, Universidad Complutense de Madrid, Madrid, España \\ Trabajo desarrollado en la Universidad del Valle Cali, Colombia
}

Introducción. Los implantes pueden ser colonizados por microorganismos de la biopelícula oral y así afectarse su salud. Entre los patógenos encontrados están los típicamente relacionados con periodontitis, como Aggregatibacter actinomycetemcomitans y Porphyromonas gingivalis, así como bacilos Gram negativos entéricos, los cuales no son clásicos de la enfermedad periodontal.

Objetivo. Determinar el perfil de las bacterias sobreinfecciosas en lesiones periimplante (mucositis o periimplantitis), en pacientes con historia de periodontitis.

Materiales y métodos. Se estudiaron 68 implantes en 55 pacientes; 49 implantes tuvieron lesión periimplante19 se consideraron estables. Se obtuvieron muestras subgingivales de implantes afectados y estables; éstas fueron sembradas en Agar MacConkey e incubadas a $37^{\circ} \mathrm{C}$ por 24 horas. Las colonias aisladas fueron identificadas con el estuche BD BBL-Cristal E/NF®.

Resultados. En 20 de los pacientes estudiados se detectaron bacterias sobreinfecciosas, pero, con mayor frecuencia en pacientes con implantes afectados $(n=15)$ que en portadores de implantes sanos $(n=5)$. La prevalencia de bacterias sobreinfecciosas en todos los implantes fue de 33,8 \% ( $n=23 / 68)$, y también fue más frecuente su aislamiento en implantes afectados $(n=17)(25 \%)$, que en estables $(n=6)$ $(8,8 \%)$. Klebsiella pneumoniae fue el microorganismo que se aisló con mayor frecuencia en todos los implantes $(n=12)$.

Conclusiones. Un tercio de los implantes estudiados presentaron organismos sobreinfecciosos. Los implantes con lesiones periimplante presentan una mayor frecuencia de bacterias sobreinfecciosas. K. pneumoniae es la especie sobreinfecciosa más frecuente en los implantes estudiados. Se presentó infección múltiple con dichas bacterias en los implantes afectados. Estos microorganismos pueden afectar la estabilidad de los implantes.

Palabras clave: bacterias Gram negativas, Enterobacteriaceae, mucositis, implantes dentales, periodontitis.

Enteric Gram negative rods and unfermented of glucose bacteria in patients with peri-implant disease

Introduction. Implants can be colonized by microorganisms from oral biofilms and may affect periimplant tissues health. Among these bacteria, pathogens typically associated with periodontitis can be found, such as Aggregatibacter actinomycetemcomitans and Porphyromonas gingivalis, as well as Gram negative enteric bacilli not typically associated with periodontal diseases.

Objective. Superinfecting bacteria were characterized from peri-implant lesions in patients with history of periodontitis.

Materials and methods. Sixty-eight implants were studied in 55 patients; the average patient age was 56 years. Forty-nine implants had peri-implant lesions and 19 were considered stable. Subgingival samples were obtained in affected and stable implants. The samples were streaked on Mac-Conkey agar and incubated at $37^{\circ} \mathrm{C}$ for 24 hours. The colonies were identified with the kit-BD BBL Crystal E/ $\mathrm{NF}$.

Results. Superinfecting organisms were detected in 20 patients--they were seen more frequently at diseased implants $(n=15)$ than at healthy implants $(n=5)$. The prevalence of superinfecting bacteria on the selected implants was $33.8 \%(n=23 / 68)$. These bacteria were more prevalent among affected implants $(n=17$ or $25 \%)$ than those with stable implants $n=6(8.8 \%)$. Klebsiella pneumoniae was the most frequent Gram negative rod detected $(n=12)$.

Conclusions. One-third of the implants had superinfecting organisms. Implants with a peri-implant lesion had a higher frequency of superinfecting bacteria. Klebsiella pneumoniae was the most common 
superinfecting organism isolated. A multiple infection caused by superinfecting bacteria was present only at diseased implants. These microbial agents potentially affect implant stability.

Key words: Gram-negative bacteria, enterobacteriaceae, mucositis, dental implants, periodontitis.

Enfermedades como la caries, la periodontitis y el trauma facial originan la pérdida de piezas dentales, que frecuentemente son reemplazadas por implantes osteointegrados. Al igual que los dientes naturales, los implantes pueden ser colonizados por microorganismos patógenos de la biopelícula oral y, de este modo, la salud del implante puede verse afectada por la presencia de dichas bacterias (1-3).

El término "periimplantitis" fue introducido en 1987 y describe una enfermedad inflamatoria destructiva del tejido óseo que forma parte de la osteointegración, lo cual lleva a la formación de la bolsa periimplante y a la pérdida del hueso de soporte marginal $(1,2)$. Se distinguen dos fases en la respuesta que se produce ante la colonización microbiana con patógenos de la superficie del implante: una inicial, que afecta al tejido blando alrededor de los implantes, denominada mucositis periimplante, y otra fase más avanzada, denominada periimplantitis, que conduce a la destrucción del tejido óseo periimplante (3-5).

Estos procesos patológicos están acompañados de un incremento en la proporción de especies patógenas asociadas con las periodontitis (3). En las periimplantitis existe un aumento del crecimiento de especies periodontopatógenas, tales como: Aggregatibacter actinomycetemcomitans, Porphyromonas gingivalis, Tannerella forsythia, Parvimonas micra, Campylobacter rectus, Fusobacterium spp., Capnocytophaga spp. y Prevotella intermedial nigrescens, entre otros organismos que, también se pueden encontrar en sitios periimplante estables, pero en bajas proporciones $(2,6)$. Los implantes estables albergan principalmente cocos Gram positivos aerobios y anaerobios (7). La microbiota presente en la cavidad oral antes de la inserción del implante y el estado periodontal de los dientes adyacentes a las localizaciones que van a recibir implantes, determinan la composición de la biopelícula del implante y, en una alta proporción, la salud del implante (8).

Correspondencia:

Adolfo Contreras, calle 4B No. 36-00, edificio 100, Cali, Colombia

Teléfono: (052) 5185680 y 518 5674; fax: (052) 5581936

adolfoco@yahoo.com

Recibido: 31/03/10; aceptado:13/09/10
Se han reportado unos microorganismos con potentes factores de virulencia y de resistencia antibióticaenperiodontitisyenlesionesperiimplante, las cuales, debido a estas características, se han denominado sobreinfecciosas. Las especies identificadas han sido cocos Gram positivos como Staphylococcus aureus y Staphylococcus epidermidis, levaduras como Candida albicans, y bacilos Gram negativos no fermentadores de la glucosa como Pseudomonas aeruginosa, así como bacterias Gram negativas entéricas pertenecientes a la familia Enterobacteriaceae como Escherichia coli, Enterobacter spp. y Klebsiella spp., $(6,9,10)$. Entre los microorganismos entéricos y no fermentadores reportados en implantes estables, se encuentran frecuencias de detección entre $8 \%$ y $17 \%(2,6,11,12)$ y, en los implantes afectados, entre $27 \%$ y $75 \%(6,11-13)$.

Estos patógenos se han asociado con infecciones de la mucosa en pacientes con enfermedades médicas, en pacientes con prótesis dentales y en adultos mayores (6). Esto puede indicar un papel oportunista de los sobreinfecciosos en las periodontitis y la periimplantitis. Para determinar la magnitud del problema en la población estudiada, se realizó un estudio descriptivo de corte transversal cuyo objetivo fue determinar el perfil de las bacterias sobreinfecciosas en lesiones periimplante y su frecuencia de detección de acuerdo con el tipo de implantes (estables o con enfermedad periimplante).

\section{Materiales y métodos}

\section{Población de estudio}

La selección de la muestra se hizo por conveniencia y se incluyeron pacientes provenientes del Servicio de Odontología de la Escuela de Odontología de la Universidad del Valle. Fueron informados del objetivo del estudio y sólo se incluyeron aquellos que voluntariamente aceptaron participar con la firma del consentimiento informado. Este estudio fue avalado por el Comité de Ética para la investigación con seres humanos de la Universidad del Valle y el protocolo cumple con la Declaración de Helsinki del 2008.

Se estudiaron 68 implantes en 55 pacientes, de los cuales, 19 implantes fueron caracterizados como estables y 49 como afectados con enfermedad 
periimplante (mucositis periimplante o periimplantitis) (cuadro 1), definida por la presencia de una profundidad de sondeo de $4 \mathrm{~mm}$ o mayor, sangrado al sondeo y pérdida radiológica de soporte óseo (en los casos de periimplantitis). Ninguno de los sujetos estudiados había recibido tratamiento periodontal o periimplante, o antibióticos en los últimos seis meses. La figura 1 representa la imagen radiográfica de dos implantes con periimplantitis.

\section{Toma de muestras microbiológicas}

Se obtuvieron muestras subgingivales de los implantes afectados y estables; en aquellos pacientes que tenían más de un implante, la muestra microbiológica fue un conjunto de los implantes de acuerdo con el grupo al que pertenecían (afectados o estables), Antes de la toma de la muestra, la placa supragingival se removió con una "cureta" (curette) estéril y el implante se aisló con rollos de algodón. Se insertaron puntas de papel de endodoncia número 35 en tres de los sitios periimplante más profundos durante 15 segundos y se transfirieron a un vial que contenía el medio de transporte VGMA III. Las muestras se remitieron al Laboratorio de Microbiología Oral y Periodontal de la Universidad del Valle.

\section{Cultivo de muestras periodontales e identificación de bacilos Gram negativos entéricos y no fermentadores}

Las muestras fueron homogenizadas con agitador por 45 segundos y se sembraron $100 \mu \mathrm{l}$ en agar MacConkey y una dilución de $10^{-1}$ e incubadas a $37^{\circ} \mathrm{C}$ en condiciones aerobias por 24 horas. Transcurrido el tiempo de incubación, se observaron las cajas inoculadas, prestando especial atención a la morfología de las colonias para identificar diferentes tipos bacterianos que pudieran corresponder a organismos sobreinfecciosas. Estas colonias se aislaron nuevamente en otra caja de agar MacConkey y se incubaron por 24 horas a $37{ }^{\circ} \mathrm{C}$. Las bacterias aisladas se identificaron con el estuche $\mathrm{BD}$ BBL-Cristal $\mathrm{E} \mathrm{NF}^{\circledR}$ para

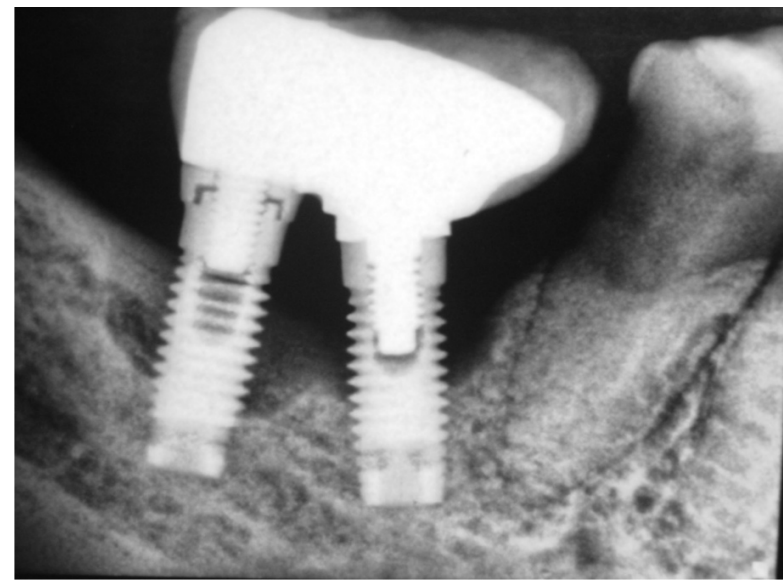

Figura 1. Imagen de radiografía digital de dos implantes mandibulares en la zona del 36 con pérdida de la osteointegración en los dos tercios más coronales de los implantes. Clínicamente, se determinaron también signos de perimplantitis, como sangrado al sondeo y formación de bolsa alrededor de los implantes

identificación de bacilos Gram negativos entéricos y no fermentadores, siguiendo las recomendaciones del fabricante, previa realización de las pruebas de oxidasa e indol.

\section{Análisis estadístico}

Se evaluaron las diferencias en la frecuencia de detección de las bacterias sobreinfecciosas entre los implantes con enfermedad periimplante y los implantes sanos por medio del test de ji al cuadrado. Se consideró una diferencia estadísticamente significativa cuando $P \leq 0,05$. Todos los datos fueron analizados utilizando el software estadístico Stata $2.0 \AA$.

\section{Resultados}

La edad promedio de la población estudiada fue de 56 años y la distribución por sexo se observa en el cuadro 1. De los pacientes estudiados, 18 $(32,7 \%)$ no tenían enfermedad periodontal, 16 $(29,1 \%)$ tenían gingivitis, $16(29,1 \%)$ presentaban periodontitis crónica y $5(9,1 \%)$ eran desdentados.

Cuadro 1. Características sociodemográficas de la población estudiada.

\begin{tabular}{|c|c|c|c|c|c|c|c|c|c|}
\hline \multirow{2}{*}{$\begin{array}{l}\text { Estado del } \\
\text { implante }\end{array}$} & \multirow{2}{*}{$\begin{array}{c}n \\
(\%)\end{array}$} & \multirow{2}{*}{$\begin{array}{c}\text { Edad } \\
\text { promedio } \\
\text { (años) (DE) }\end{array}$} & \multicolumn{2}{|c|}{ Sexo } & \multicolumn{4}{|c|}{ Localización } & \multirow{2}{*}{$\begin{array}{c}\text { Promedio } \\
\text { tiempo de } \\
\text { carga } \\
\text { meses }\end{array}$} \\
\hline & & & $F(\%)$ & M (\%) & Maxilar & $\begin{array}{c}\bar{X} \text { PD } \\
(\mathrm{mm})\end{array}$ & Mandibular & $\begin{array}{c}\bar{X} \text { PD } \\
(\mathrm{mm})\end{array}$ & \\
\hline Estable & $\begin{array}{c}19 \\
(27,9)\end{array}$ & $57,1(13,9)$ & $\begin{array}{c}8 \\
(66,7)\end{array}$ & $\begin{array}{c}4 \\
(33,3)\end{array}$ & $45,5 \%$ & 3,3 & $54,5 \%$ & 3,56 & 44,96 \\
\hline Afectado & $\begin{array}{c}49 \\
(72,1)\end{array}$ & $56,3(14,3)$ & $\begin{array}{c}30 \\
(69,8)\end{array}$ & $\begin{array}{c}13 \\
(30,2)\end{array}$ & $60,7 \%$ & 4,54 & $39,3 \%$ & 4,42 & 46,08 \\
\hline
\end{tabular}


La prevalencia de bacterias sobreinfecciosas en implantes fue de 33,8 \% (23/68). El aislamiento de estos microorganismos fue más frecuente en los implantes afectados, en comparación con los implantes estables: $\mathrm{n}=17(25 \%)$ Vs. $\mathrm{n}=6(8,8$ $\%)$, respectivamente. Klebsiella pneumoniae fue el único agente sobreinfeccioso aislado en los implantes estables y, al mismo tiempo, fue el más aislado en los implantes afectados $(n=9)$, seguido por Burkholderia cepacia $(n=4)$, Klebsiella oxytoca $(n=2)$ y $P$. aeruginosa, Enterobacter gergoviae, $E$. coli, Enterobacter cloacae y Stenotrophomonas maltophilia ( $\mathrm{n}=1$ ) (cuadro 2). El rango de los conteos microbianos de microorganismos sobreinfecciosos en unidades formadoras de colonias (UFC) en el medio selectivo fue entre 1 UFC y 300 UFC, y su promedio fue de 55,8 UFC.

Los implantes afectados presentaron infecciones multibacterianas y se localizaron con mayor frecuencia en el maxilar superior.

\section{Discusión}

La primera aproximación microbiológica para determinar la etiología de las lesiones periimplante fue realizada por Rams et al. en 1984, quienes observaron una alta proporción de espiroquetas por microscopía electrónica en muestras tomadas alrededor de implantes con evidencia de lesión periimplante (14). En 1987, Mombelli et al. cultivaron e identificaron la microbiota de lesiones periimplante y la compararon con la proveniente de implantes sanos de los mismos individuos y de individuos que sólo tuvieron implantes estables. El $41 \%$ de los microorganismos cultivados a partir de las lesiones periimplante fueron bacilos Gram negativos anaerobios, como Fusobacterium spp. y $P$. intermedia; mientras que los implantes estables presentaron bajos recuentos bacterianos, aislándose principalmente cocos Gram positivos (15). En ese mismo año, Nakou et al, determinaron la colonización temprana en implantes de hidroxiapatita en cinco pacientes desdentados y encontraron espiroquetas en la placa supragingival y Actinomyces odontolyticus, A. actinomycetemcomitans, Eikenella corrodens y Capnocytphaga sputigena (16). Estos dos hallazgos permitieron concluir que la enfermedad periimplante es una enfermedad específica para cada sitio que conserva los patrones microbiológicos típicos de la enfermedad periodontal.

Al final de los años 1980, otros estudios identificaron especies bacterianas no relacionadas típicamente con enfermedad periodontal, tales como los bacilos Gram negativos entéricos, los cuales corresponden a microbiota residente de la luz del intestino distal, incluyendo la familia Enterobacteriaceae, y los géneros Acinetobacter spp. y Pseudomonas spp., entre otros. Estos microorganismos fueron denominados sobreinfecciosos por Helovuo et al., quienes demostraron que el tratamiento con antibióticos de amplio espectro en pacientes con periodontitis, como parte de la terapia periodontal, aumenta la prevalencia de estos microorganismos como oportunistas (17). Slots et al., en 1988, identificaron diferentes especies de la familia Enterobacteriaceae y Pseudomonas spp. en 500 adultos con periodontitis grave, con una frecuencia de 10,2 \% (51/500); se aislaron, principalmente, E. cloacae, Enterobacter agglomerans, Proteus mirabilis, K. pneumoniae y K. oxytoca (18).

En relación con los implantes, diversos estudios han identificado microorganismos sobreinfecciosos en lesiones periimplante, con frecuencias similares a las encontradas en dientes de pacientes con periodontitis $(6,11-13)$.

Cuadro 2. Frecuencia de microorganismos sobreinfecciosos en los implantes estudiados.

\begin{tabular}{|c|c|c|c|c|c|c|c|}
\hline \multirow[t]{2}{*}{ Microorganismo sobreinfeccioso } & \multicolumn{2}{|c|}{$\begin{array}{l}\text { Frecuencia } \\
\text { en total de } \\
\text { implantes }\end{array}$} & \multicolumn{2}{|c|}{$\begin{array}{c}\text { Frecuencia } \\
\text { en implantes } \\
\text { afectados }\end{array}$} & \multicolumn{2}{|c|}{$\begin{array}{c}\text { Frecuencia } \\
\text { en implantes } \\
\text { estables }\end{array}$} & \multirow[t]{2}{*}{ Valor de $\mathbf{P}^{*}$} \\
\hline & $\mathbf{n}$ & $\%$ & $\mathbf{n}$ & $\%$ & $\mathbf{n}$ & $\%$ & \\
\hline Klebsiella pneumoniae & 12 & 17,6 & 7 & 14,3 & 5 & 7,4 & 0,243 \\
\hline Pseudomonas aeruginosa & 1 & 1,5 & 1 & 1,5 & 0 & 0 & 0,530 \\
\hline Enterobacter gergoviae & 1 & 1,5 & 1 & 1,5 & 0 & 0 & 0,530 \\
\hline Escherichia coli & 1 & 1,5 & 1 & 1,5 & 0 & 0 & 0,530 \\
\hline Enterobacter cloacae & 1 & 1,5 & 0 & 0 & 1 & 1,5 & 0,106 \\
\hline Klebsiella oxytoca & 2 & 2,9 & 2 & 2,9 & 0 & 0 & 0,371 \\
\hline Burkholderia cepacea & 4 & 5,9 & 4 & 5,9 & 0 & 0 & 0,199 \\
\hline Stenotrophomonas maltophilia & 1 & 1,5 & 1 & 1,5 & 0 & 0 & 0,530 \\
\hline
\end{tabular}

*Ji al cuadrado 
La prevalencia de organismos sobreinfecciosos en el total de implantes analizados en el presente estudio, fue de $33,8 \%$, y fueron más frecuentes en los implantes con lesiones periimplante $(25 \%)$ en comparación con los implantes estables $(8,8$ $\%)(p=0,08$, ji al cuadrado). Aunque las diferencias no fueron estadísticamente significativas, estos resultados sugieren una frecuencia mayor de detección de sobreinfecciosos en lesiones periimplante (cuadro 2).

Klebsiella pneumoniae fue la especie más frecuentemente identificada en implantes afectados y estables, organismo que fue detectable en el aparato respiratorio y en el tubo digestivo en menos de $5 \%$ de las personas sanas. Es responsable de $1 \%$ de las neumonías bacterianas y, en ocasiones, origina infecciones urinarias y bacteriemias en casos de compromiso inmunitario. Los principales factores de virulencia de $K$. pneumoniae es el lipolisacárido que estimula en el organismo la producción de IL-1 beta y FNTa, ambas citocinas implicadas en la destrucción de los tejidos periodontales. Los lipolisacáridos de los organismos sobreinfecciosos han demostrado una mayor capacidad antigénica y una mayor virulencia que los producidos por los típicos organismos anaerobios Gram negativos implicados en periodontitis (19).

El otro microorganismo que se identificó solamente en los casos de implantes afectados fue $B$. cepacia, que se considera un microorganismo ambiental pero ha sido reportado en infecciones hospitalarias por la multirresistencia a los antibióticos que posee. Este microorganismo es causante de una importante morbilidad y mortalidad en casos de fibrosis quística (20).

Otros organismos sobreinfecciosos (cuadro 2) que aparecieron en los implantes afectados tuvieron bajas frecuencias de detección, lo que permite suponer que forman parte de una microbiota transitoria, pero, por sus importantes factores de virulencia, pueden igualmente afectar la salud de los implantes.

El tratamiento de las infecciones periimplante está dirigido a eliminar o suprimir la microbiota patógena alrededor del implante, para lo que se han empleado diversos protocolos que consideran los siguientes aspectos:

a) control efectivo de la placa por parte del paciente, con una correcta educación en técnicas de higiene oral;

b) desbridamiento subgingival a campo cerrado, con el uso de antibióticos o antimicrobianos o sin ellos; c) cirugía de resección periimplante para retirar los tejidos infectados y armonizar la arquitectura ósea, con el fin de reducir la profundidad de la bolsa periimplante y facilitar la limpieza de la placa dental por el paciente; $y$

d) cirugía regenerativa para tratar de rellenar los defectos óseos alrededor de los implantes o recuperar los tejidos perdidos con materiales aloplásticos o hueso autólogo (21).

El tratamiento de las afecciones periimplante es todavía controversial, al no haber evidencia que soporte el uso preferencial de algún protocolo, y los estudios realizados presentan algunas limitaciones metodológicas (21). Sin embargo, los antimicrobianos actualmente usados no consideran el control de los microorganismos sobreinfecciosos. Dichas bacterias pueden permanecer en los tejidos periimplante durante un mayor tiempo después de un tratamiento convencional de reducción de la profundidad de la bolsa o colocación de injertos óseos, por las ventajas ecológicas que poseen, como son: la aerotolerancia, menores demandas nutricionales y capacidad de residir en reservorios extraperiodontales (12). En general, se desconoce la dinámica de colonización, las proporciones de los microorganismos sobreinfecciosos en relación con la microbiota subgingival en enfermedad periimplante y su capacidad de organización en diversos nichos orales. Así, resulta importante determinar en futuros estudios la proporción de dichos microorganismos en relación con la microbiota periimplante.

Un $30 \%$ de los implantes estudiados presentaron organismos sobreinfecciosos. Klebsiella pneumoniae fue la especie sobreinfecciosa cultivada con mayor frecuencia; por dicha razón, consideramos que se requieren nuevos estudios que permitan determinar el papel de los sobreinfecciosos en la etiología y patogénesis de la enfermedad periimplante, así como su impacto en la salud de los implantes.

\section{Conflicto de intereses}

Los autores declaran que no existe conflicto de interés.

\section{Financiación}

Laboratorio de Microbiología Oral y Periodontal, Departamento de Microbiología, Facultad de Salud, Universidad del Valle y a Colciencias por la financiación de las jóvenes investigadoras Jessika Consuegra y Sonia Jakeline Gutiérrez, en el marco del programa "Jóvenes Investigadores e Innovadores Virginia Gutiérrez de Pineda". 


\section{Referencias}

1. Meffert RM, Block JM. What is osseointegration? Int J Periodont Rest Dent. 1987;4:3-16.

2. Quirynen M, Teughels. Microbiologically compromised patients and impact on oral implants. Periodontol 2000. 2003;33:119-28.

3. López L. Infecciones relacionadas con los implantes dentarios. Enferm Infecc Microbiol Clin. 2008;26:589-92.

4. Jovanovic S. The management of peri-implant breakdown around functioning osseointegrated dental implants. J Periodontol. 1993;64:1176-83.

5. Heitz-Mayfield LJ. Peri-implant diseases: Diagnosis and risk indicators. J Clin Periodontol. 2008;35(Suppl.8):292304.

6. Leonhardt A, Renvert S, Dahlén G. Microbial findings at failing implants. Clin Oral Implants Res. 1999;10:339-45.

7. George K, Zafiropoulos GG, Murat Y, Hubertus S, Nisengard RJ. Clinical and microbiological status of osseointegrated implants. J Periodontol. 1994;65:766-70.

8. Mombelli A, Buseer D, Lang NP. Colonization of osseointegrated titanium implants in edentulous patients. Oral Microbiol Immunol. 1998;3:113-20.

9. Betancourt M, Arce R, Botero J, Jaramillo A, Cruz C, Contreras A. Microorganismos poco usuales en surcos y bolsas periodontales. Colombia Médica. 2006;37:6-14.

10. Pye AD, Lockhart DE, Dawson MP, Murray CA, Smith AJ. A review of dental implants and infection. J Hosp Infect. 2009;72:104-10.

11. Mombelli A. Microbiological features of stable osseointegrated implants used as abutments for overdentures. Clin Oral Implants Res. 1990;1:1-7.

12. Botero JE, González AM, Mercado RA, Olave G, Contreras A. Subgingival microbiota in peri-implant mucosa lesions and adjacent teeth in partially endentulous patients. J Periodontol. 2005;76:1490-5.

13. Alcoforado GA, Rams TE, Feik D, Slots J. Microbial aspects of failing osseointegrated dental implants in humans. J Parodontol. 1991;10:11-8.

14. Rams TE, Link CC. Microbiology of failing dental implants in humans: Electron microscopic observations. J Oral Implantol. 1983;11:93-100.

15. Mombelli A, van Oosten MAC, Schurch E, Lang NP. The microbiota associated with successful or failing osseointegrated titanium implants. Oral Microbiol Immunol. 1987;2:145-51.

16. Nakou M, Mikxs FH, Oosterwaal PJ, Kruijsen JC. Early colonization of permucosal implants in edentulous patients. J Dent Res. 1987;66:1654-57.

17. Helovuo H, Hakkarainen K, Paunio K. Changes in the prevalence of subgingival enteric rods, staphylococci and yeasts after treatment with penicillin and erythromycin. Oral Microbiol Immunol. 1993;13:390-3.

18. Slots J, Rams T, Listgarten M. Yeast, enteric rods and pseudomonads in the subgingival flora of severe adult periodontitis. Oral Microbiol Immunol. 1990;5:29-32.

19. Arakawa Y. Genomic organization and regulation of $\mathrm{cps}$ cluster that is involved in synthesis of capsular polysaccharide as a virulence factor of Klebsiella pneumonia. Nippon Saikingaku Zasshi. 1994;49:455-63.

20. Lynch JP. Burkholderia cepacia complex: Impact on the cystic fibrosis lung lesion. Semin Respir Crit Care Med. 2009;30:596-610.

21. Claffey N, Clarke E, Polyzois I, Renvert S. Surgical treatment of periimplantitis. J Clin Periodontol. 2008;35(Suppl.8):316-32. 